\title{
NOMOGRAPHIC FUNCTIONS ARE NOWHERE DENSE
}

\author{
R. CREIGHTON BUCK
}

ABstract. A function $f$ of $n$ variables is nomographic if it can be represented in the format

$$
f\left(x_{1}, \ldots, x_{n}\right)=h\left(\phi_{1}\left(x_{1}\right)+\cdots+\phi_{n}\left(x_{n}\right)\right)
$$

where the $\phi_{i}$ and $h$ are continuous. Every continuous function of $n$ variables has a representation as a sum of not more than $2 n+1$ nomographic functions [9]. This paper gives a constructive proof that the nomographic functions form a nowhere dense subset of the space $C\left[I^{n}\right]$.

Let $I=[-1,1]$ and $I^{k}=I \times I \times \cdots \times I$. The class $\mathcal{N}^{k}$ of nomographic functions of $k$ variables are those that on $I^{k}$ have a representation in the special format

$$
f\left(x_{1}, x_{2}, \ldots, x_{k}\right)=h\left(\phi_{1}\left(x_{1}\right)+\phi_{2}\left(x_{2}\right)+\cdots+\phi_{k}\left(x_{k}\right)\right)
$$

where the $\phi_{k}$ and $h$ are real-valued continuous functions on $-\infty<t<\infty$. Interest in $\mathcal{N}^{k}$ revived when Kolmogorov used these functions in 1957 to settle Hilbert's 13th problem by showing that every continuous function on $I^{k}$ could be written as the sum of $2 k+1$ functions from $\mathcal{N}^{k}$. (See $[9,10]$.)

Formula (1) makes it natural to conjecture that $\mathcal{N}^{k}$ is a sparse subset of the space $C\left[I^{k}\right]$ of all real continuous functions on $I^{k}$, with the usual norm $\|g\|=\max |g(p)|$, as suggested in [2]. This does not conflict with the Kolmogorov result; for example, $[0,1]$ is the algebraic sum of two copies of a nowhere dense subset $E$. That $\mathcal{N}^{k}$ should be sparse is more evident when smoothness is required. If $f \in \mathcal{N}^{2}$ has component functions $h, \phi_{1}$, and $\phi_{2}$ which are in $C^{\prime \prime \prime}$, then $f$ must satisfy a third order PDE that is characteristic for $\mathcal{N}^{2}$. (See [4].) In addition, nowhere denseness is known to follow smoothness in a number of other studies of superposition classes. (See $[6,7,8]$.) In the present paper, we present a direct constructive proof that $\mathcal{N}^{k}$ is nowhere dense in $C\left[I^{k}\right]$, with no differentiability restrictions on $h$ or $\phi_{j}$; indeed, we do not require that $h$ be continuous. We remark that $\mathcal{N}^{2}$ is not uniformly closed. (See $[1$ or 3$]$.)

We prove a more general theorem, and then verify later that the requisite property is shared by the class $\mathcal{N}^{k}$.

Let $D$ be a compact set in $R^{n}$ with nonvoid interior, and $C[D]$ be the Banach space of real-valued continuous functions on $D$, with the uniform convergence norm $\|F\|_{D}=\max _{p \in D}|F(p)|$. Let $₹$ be a subset of $C[D]$ which we will prove is nowhere dense. The key requirement we need is the existence of special functions in $C[D]$ that fail to belong locally to the closure of $₹$.

Received by the editors August 10, 1981.

1980 Mathematics Subject Classification. Primary 41A30, 26 A72.

Key words and phrases. Superpositions, Hilbert, nowhere dense.

(c) 1982 American Mathematical Society 
THEOREM 1. Suppose that there is a point $p_{0}$ interior to $D$ such that for any real $c$, there exists $g \in C[D]$ with $g\left(p_{0}\right)=c$ such that for every compact neighborhood $V$ of $p_{0}$

$$
\inf _{f \in \mathcal{F}}\|f-g\|_{V}>0 .
$$

Then, $\mathcal{F}$ is nowhere dense in $C[D]$.

ProOF. Let $U$ be any nonempty open set in $C[D]$; we will produce another nonempty open set $U_{1} \subset U$ disjoint from $₹$. Choose $G_{0} \in U$ and $r>0$ such that $\left\|G-G_{0}\right\|<r$ implies $G \in U$. Let $c=G_{0}\left(p_{0}\right)$, and then use the hypothesis to select a special function $g$ in $C[D]$ obeying (2). Let $B$ be a closed ball in $D$, centered at $p_{0}$, such that

$$
\left|G_{0}(p)-c\right|<\frac{r}{3} \text { for all } p \in B,
$$

and choose a smaller ball $B_{0}$, also centered at $p_{0}$, such that

$$
|g(p)-c|<\frac{r}{3}, \quad p \in B_{0} .
$$

Construct a continuous function $G_{1}$ defined on $B$ such that $G_{1}(p)=G_{0}(p)$ for $p$ on the boundary of $B, G_{1}(p)=g(p)$ on $B_{0}$, and obeying $\left|G_{1}(p)-c\right|<{ }_{3}^{r}$ on $B$. Then, extend $G_{1}$ to all of $D$ by setting it equal to $G_{0}$ off $B$. Observe that $G_{1}$ is in $C[D]$ and agrees with $G_{0}$ except on a small neighborhood of $p_{0}$ where it has been modified to agree with the special function $g$ locally. If $p \in B$, we have

$$
\begin{aligned}
\left|G_{1}(p)-G_{0}(p)\right| & \leq\left|G_{1}(p)-c\right|+\left|c-G_{0}(p)\right| \\
& \leq \frac{r}{3}+\frac{r}{3}<r .
\end{aligned}
$$

Accordingly, $\left\|G_{1}-G_{0}\right\|_{D}<r$ and $G_{1} \in U$.

By (2), choose $\delta>0$ so that $\|f-g\|_{B_{0}}>\delta$ for all $f \in \mathcal{F}$, and take $U_{1}=$ \{all $F \in U$ with $\left.\left\|F-G_{1}\right\|_{D}<\frac{\delta}{2}\right\}$. Then, if $F \in U_{1}$ and $f \in \mathcal{F}$,

$$
\begin{aligned}
\|f-F\|_{D} & \geq\|f-g\|_{B_{0}}-\left\|F-G_{1}\right\|_{B_{0}} \\
& \geq \delta-\frac{\delta}{2}=\frac{\delta}{2} .
\end{aligned}
$$

Thus, $\mp$ is nowhere dense in $C[D]$.

We now use this to show that $\mathcal{N}^{2}$ is nowhere dense in $C\left[I^{2}\right]$. Take $p_{0}=(0,0)$; since $\mathcal{N}^{2}$ is closed under addition of constants, we do not need to retain the condition in (2) that $g\left(p_{0}\right)=c$. For any $r>0$, let $V_{r}$ be the compact neighborhood of $p_{0}$ consisting of those $(x, y)$ with $|x| \leq r,|y| \leq r$.

THEOREM 2. The special function $g(x, y)=x^{2}+x y+y^{2}+2 x+y$ has the property that

$$
r^{2}>\inf _{f \in \mathcal{N}^{2}}\|f-g\|_{V_{r}}>r^{3} / 10
$$

for all $r<.01$.

We begin the proof of this by quoting one of the characterization theorems for nomographic functions obtained in [3], modifying it to match the notation and needs of the present paper. (See Theorem 12, p. 293.) 
Let $g$ be of class $C^{\prime}$ on the set $V_{r}$, and suppose that $g_{x}$ and $g_{y}$ are bounded below by $\sigma>0$. Let $\epsilon<r \sigma / 12$ and suppose that the distance in the space $C\left[V_{r}\right]$ between $g$ and the set $\mathcal{N}^{2}$ is less than $\epsilon$. Then, one of the following systems of inequalities must be solvable.

(i) For some choice of $x_{i}$ in $[-r, r]$,

$$
\begin{aligned}
\left|g\left(x_{1},-r\right)-g(-r, 0)\right| & <2 \epsilon, \\
\left|g\left(x_{2},-r\right)-g(-r, r)\right| & <2 \epsilon, \\
\left|g\left(x_{1}, r\right)-g\left(x_{2}, 0\right)\right| & <2 \epsilon .
\end{aligned}
$$

(ii) For some choice of $y_{i}$ in $[-r, r]$,

$$
\begin{array}{r}
\left|g\left(-r, y_{1}\right)-g(0,-r)\right|<2 \epsilon, \\
\left|g\left(-r, y_{2}\right)-g(r,-r)\right|<2 \epsilon, \\
\left|g\left(r, y_{1}\right)-g\left(0, y_{2}\right)\right|<2 \epsilon .
\end{array}
$$

If we apply this general result to the special function $g(x, y)$ in Theorem 2, and assume that the distance from $g$ to $\mathcal{N}^{2}$ is less than $\epsilon$, then after putting $x_{i}=r s_{i}$ and $y_{i}=r t_{i}$, we have either there are $s_{i}$ with $\left|s_{i}\right| \leq 1$ and

$$
\begin{aligned}
\left|2 s_{1}+1+\left(s_{1}^{2}-s_{1}\right) r\right| & <2 \epsilon / r, \\
\left|2 s_{2}+\left(s_{2}^{2}-s_{2}\right) r\right| & <2 \epsilon / r, \\
\left|2 s_{1}-2 s_{2}+1+\left(s_{1}^{2}-s_{2}^{2}+s_{1}+1\right) r\right| & <2 \epsilon / r ;
\end{aligned}
$$

or there exist $t_{i}$ with $\left|t_{i}\right| \leq 1$ such that

$$
\begin{aligned}
\left|t_{1}-1+\left(t_{1}^{2}-t_{1}\right) r\right| & <2 \epsilon / r, \\
\left|t_{2}-3+\left(t_{2}^{2}-t_{2}\right) r\right| & <2 \epsilon / r, \\
\left|t_{1}-t_{2}+2+\left(t_{1}^{2}-t_{2}^{2}+t_{1}+1\right) r\right| & <2 \epsilon / r .
\end{aligned}
$$

We now show that if $\epsilon=r^{3} / 10$ and $r<.01$, then neither (9) nor (10) can be satisfied. For (10) this is immediate, since the second inequality in (10) implies that

$$
\left|t_{2}-3\right| \leq 2 r+r^{2} / 5 \text {, }
$$

contradicting $\left|t_{1}\right| \leq 1$. To show that (9) also fails, set

$$
\begin{gathered}
A=2 s_{1}+1+\left(s_{1}^{2}-s_{1}\right) r, \quad B=2 s_{2}+\left(s_{2}^{2}-s_{2}\right) r, \\
C=2 s_{1}-2 s_{2}+1+\left(s_{1}^{2}-s_{2}^{2}+s_{1}+1\right) r,
\end{gathered}
$$

so that the inequalities in (10) become $|A|<r^{2} / 5,|B|<r^{2} / 5,|C|<r^{2} / 5$. The first of these implies $\left|2 s_{1}+1\right| \leq 2 r+r^{2} / 5<3 r$, and hence that $s_{1}=-\frac{1}{2}+$ ar where $|a| \leq \frac{3}{2}$. Substituting this into $A$, we have $\left|2 a+\frac{3}{4}\right| \leq 3 r+\frac{r}{5}+\frac{9}{4} r^{2}<4 r$ and conclude that $s_{1}=-\frac{1}{2}-\frac{3}{8} r+b r^{2}$ where $|b| \leq 2$. In a similar way, $|B|<r^{2} / 5$ implies that $s_{2}=c r^{2}$ where $|c| \leq 1$. Finally, since $C=A-B+\left(2 s_{1}+1-s_{2}\right) r$, we find $\left|2 s_{1}+1-s_{2}\right| \leq \frac{3}{5} r$ and using the values obtained for $s_{1}$ and $s_{2}$, we finally obtain

which clearly cannot hold if $r<.01$.

$$
\left|-\frac{3}{4}+(2 b-c) r\right| \leq \frac{3}{5}
$$

To obtain the left side of (6), we merely observe that the special function $f_{0}(x, y)=\left(x+\frac{1}{2} y+1\right)^{2}-1$ belongs to $\mathcal{N}^{2}$ and obeys $\left\|f_{0}-g\right\|_{V_{r}}<r^{2}$. 
Having proved Theorem 2, we invoke Theorem 1 and obtain

COROLLARY. $\mathcal{N}^{2}$ is a nowhere dense subset of $C\left[I^{2}\right]$.

To show now that this is also true of the class $\mathcal{N}^{k}$ in the space $C\left[I^{k}\right]$, we prove that one does not obtain a better nomographic approximation to the special function $g$ given in Theorem 2 by using nomographic functions of $k$ variables for any $k>2$.

THEOREM 3. If $g$ is any continuous function of $\left(x_{1}, x_{2}\right)$ then

$$
\inf _{f \in \mathcal{N}^{k}}\|f-g\|_{I^{k}}=\inf _{f * \mathcal{N}^{2}}\left\|f^{*}-g\right\|_{I^{2}} .
$$

Proof. Let $d$ be the distance in $C\left[I^{k}\right]$ from $g$ to $\mathcal{N}^{k}$. Given $\delta>0$, choose $f_{0} \in \mathcal{N}^{k}$ so that

$$
d_{0}=\left\|f_{0}-g\right\|_{I^{k}}<d+\delta .
$$

Choose $\bar{x}=\left(\bar{x}_{1}, \bar{x}_{2}, \ldots, \bar{x}_{k}\right)$ in $I^{k}$ so that

$$
d_{0}=\left|f_{0}(\bar{x})-g(\bar{x})\right|=\left|h\left(\phi_{1}\left(\bar{x}_{1}\right)+\phi_{2}\left(\bar{x}_{2}\right)+\cdots+\phi_{k}\left(\bar{x}_{k}\right)\right)-g(\bar{x})\right| .
$$

Define a function $\psi$ on $I$ by

$$
\psi(s)=\phi_{2}(s)+\phi_{3}\left(\bar{x}_{3}\right)+\cdots+\phi_{k}\left(\bar{x}_{k}\right),
$$

and then set $f^{*}\left(x_{1}, x_{2}\right)=h\left(\phi_{1}\left(x_{1}\right)+\psi\left(x_{2}\right)\right)$. It is then clear that

$$
\left|f^{*}\left(\bar{x}_{1}, \bar{x}_{2}\right)-g\left(\bar{x}_{1}, \bar{x}_{2}\right)\right|=d_{0}
$$

while for any $\left(x_{1}, x_{2}\right)$ in $I^{2}$,

$$
\left|f^{*}\left(x_{1}, x_{2}\right)-g\left(x_{1}, x_{2}\right)\right|=\left|f_{0}\left(x_{1}, x_{2}, \bar{x}_{3}, \ldots, \bar{x}_{k}\right)-g\left(x_{1}, x_{2}\right)\right| \leq\left\|f_{0}-g\right\|_{I^{k}} .
$$

Accordingly, $\left\|f^{*}-g\right\|_{I^{2}}=\left\|f_{0}-g\right\|_{I^{k}}<d+\delta$, holding for every $\delta>0$. This proves one half of (11); the rest follows since $\mathcal{N}^{2}$ is part of $\mathcal{N}^{k}$.

While five copies of $\mathcal{N}^{2}$ are enough to give $C\left[I^{2}\right]$ as their algebraic sum, it is known that four will not suffice. (See [5].) It would be of interest to know if the sum of four copies of $\mathcal{N}^{2}$ is perhaps also nowhere dense in $C\left[I^{2}\right]$. The argument we have used here will not work since the special function $g(x, y)$ used in Theorem 2 in fact is already a member of $\mathcal{N}^{2}+\mathcal{N}^{2}$. Indeed,

$$
g(x, y)=\exp (\log (x+2)+\log (y+3))+\left(x^{2}-x+y^{2}-y-6\right) .
$$

\section{REFERENCES}

1. V. I. Arnold, On the representability of functions of two variables in the form $\chi(\phi(x)+\psi(y))$, Uspehi Mat. Nauk 12 (1957), 119-121. (Russian)

2. - Some questions on approximation and representation of functions, Proc. Internat. Congr. Math., 1958, Cambridge Univ. Press, New York, 1960.

3. R. C. Buck, Approximate complexity and functional representation, J. Math. Anal. Appl. 70 (1979), 280-298.

4. - Characterization of classes of functions, Amer. Math. Monthly 88 (1981), 139-142.

5. Raouf Doss, On the representation of the continuous functions of two variables by means of addition and continuous functions of one variable, Colloq. Math. 10 (1963), 249-259.

6. B. L. Fridman, Nowhere denseness of the space of linear superpositions of functions of several variables, Izv. Akad. Nauk SSSR Ser. Mat. 36 (1972), 814-846. (Russian)

7. G. M. Henkin, Linear superpositions of continuous differentiable functions, Dokl. Akad. Nauk SSSR 157 (1964), 288-290. (Russian) 
8. Robert Kaufman, Linear superpositions of smooth functions, Proc. Amer. Math. Soc. 46 (1974), 360-362.

9. A. N. Kolmogorov, On the representation of continuous functions of several variables by superpositioins of continuous functions of one variable, and addition, Dokl. Akad. Nauk SSSR 114 (1957), 953-957. (Russian)

10. G. G. Lorentz, The 13th problem of Hilbert, Mathematical Developments Arising from Hilbert's Problems, Proc. Sympos. Pure Math., vol. 28, Amer. Math. Soc., Providence, R. I., 1976.

11. David Sprecher, A survey of solved and unsolved problems in the superposition of functions, $J$. Approx. Theory 6 (1972), 123-134.

DEPARTMENT OF MATHEMATICS, UNIVERSITy OF WISCONSIN, MADISON, WisCONSIN 53706 